(2) Open Access Full Text Article

\title{
Neutrophil/lymphocyte ratio and mean platelet volume in central serous chorioretinopathy
}

\author{
This article was published in the following Dove Press journal: \\ Therapeutics and Clinical Risk Management \\ 28 July 2017 \\ Number of times this article has been viewed
}

\author{
Muhammet Kazim Erol' \\ Ayse Balkarli² \\ Ozgur Yucel' \\ Yusuf Akar ${ }^{3}$ \\ Berna Dogan' \\ Elcin Suren' \\ 'Department of Ophthalmology, \\ ${ }^{2}$ Department of Internal Medicine, \\ Division of Rheumatology, Antalya \\ Education and Research Hospital, \\ ${ }^{3}$ Department of Ophthalmology, \\ Akdeniz University Medical Faculty, \\ Antalya, Turkey
}

\begin{abstract}
Purpose: This study aimed to evaluate mean platelet volume (MPV) and neutrophil/lymphocyte ratio (NLR) values and their relationship with clinical findings in patients with central serous chorioretinopathy (CSCR).

Methods: Overall, 87 patients fulfilling inclusion criteria and 320 age- and sex-matched healthy individuals as controls were included in the study. The CSCR patients $(n=87)$ were classified into 2 groups as acute CSCR (group 1, n=43) and chronic CSCR (group 2, n=44).

Results: It was found that NLR $(P<0.05)$ and C-reactive protein $(\mathrm{CRP})(P<0.05)$ values were higher in acute CSCR group than the other groups. MPV value was found to be higher in chronic CSCR group than the other groups $(P<0.001)$.

Conclusion: It seems that neutrophils play a major role in acute CSCR while platelets are involved in progression to chronic CSCR. Larger, prospective studies are needed on this topic. Keywords: central serous chorioretinopathy, mean platelet volume, neutrophil/lymphocyte ratio
\end{abstract}

\section{Introduction}

Central serous chorioretinopathy (CSCR) is a retinal disorder known to cause visual impairment, and is characterized by serous detachment of neurosensory retina. ${ }^{1,2}$ It typically affects middle-aged men. Although several risk factors have been identified in the pathogenesis of CSCR, the exact pathophysiological mechanism remains unclear. ${ }^{3-6}$ Current advances in retinal imaging modalities, particularly in indocyanine green angiography, improve our understanding of CSCR pathophysiology with interest in focusing on hemodynamics at choroidal level. CSCR primarily involves choroidal circulation, resulting in multiple areas of vascular choroidal hyperpermeability. ${ }^{1,7,8}$ There are further abnormalities in choroidal perfusion or vascular endothelial growth factor (VEGF). ${ }^{9}$ VEGF was formerly known as "vascular hyperpermeability factor", and it is also known that IL-6 and IL-8 have profound effects of vascular hyperpermeability. ${ }^{10-12}$ Thus, it has been thought that angiogenic cytokines such as VEGF, IL-6, and IL-8 as well as other proinflammatory cytokines might be increased in patients with CSCR. This idea has become the focus of interest and many studies have been performed on cytokines and oxidative stress. ${ }^{1,13-18}$ However, results are controversial. Oxidative stress and resultant hypoxia may cause an increase in epinephrine levels, leading to choroidal vasoconstriction and disturbances in choroidal circulation, which are well-described in CSCR. ${ }^{16}$

Pathophysiology of CSCR has not been fully elucidated. However, there are some well-known risk factors including male gender, cortisol levels, pregnancy, hypertension, stress, smoking, abnormal coagulation, platelet aggregation, sleep apnea syndrome,
Department of Ophthalmology, Antalya

Education and Research Hospital,

Varlık Mah., Kazim Karabekir Caddesi,

07100 Antalya, Turkey

Tel +90242 2494400

Email bernadoga3@hotmail.com
Therapeutics and Clinical Risk Management 2017:13 945-950

945

Dovepress f $y$ in 0

http://dx.doi.org/10.2147/TCRM.S138581 (c) 1 (1) (8) 2017 Erol et al. This work is published and licensed by Dove Medical Press Limited. The full terms of this license are available at https://www.dovepress.com/terms.php

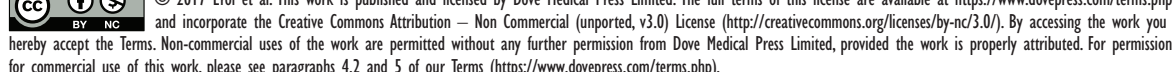


and type A personality trait. In a study by Iijima et al, it was indicated that choroidal circulation abnormality observed in CSCR was comparable to the angiographic abnormality seen in the retinal circulation of branch retinal vein occlusion. ${ }^{17}$ Sogutlu Sari et al proposed the hypothesis that choroidal hyperpermeability develops because of thrombotic occlusion in choroidal vessels and impaired fibrinolysis. ${ }^{18}$ The role of inflammation has not been elucidated in the etiopathogenesis of CSCR; however, it is still the focus of interest in this field. In the literature, CSCR has been suggested as a systemic inflammatory disease.

In recent years, the reports have concluded that neutrophil/ lymphocyte ratio (NLR) and mean platelet volume (MPV) are indicators for systemic inflammation. ${ }^{19-23}$ Mean thrombocyte size is reported as MPV in complete blood count (CBC). MPV is directly associated with platelet activity. Larger platelets are more active than smaller platelets in metabolic and enzymatic manner and also have higher potential of thrombogenicity. ${ }^{24-26}$ NLR can be readily estimated from $\mathrm{CBC}$. In addition, it has been reported that these parameters may have prognostic implications in some diseases. ${ }^{27,28}$

To the best of our knowledge, in the literature, the only study assessing MPV in patients with CSCR was conducted by Dursun et al. ${ }^{29}$ However, there is no study assessing MPV and NLR in patients with CSCR in the literature. In this study, it was aimed to evaluate MPV and NLR values and their relationship with clinical findings in patients with CSCR.

\section{Materials and methods}

\section{Patients}

The study protocol was approved by the local Ethics Committee of Antalya Education and Research Hospital and conducted in accordance with the Declaration of Helsinki. Prior to initiation, all subjects signed a detailed written consent form to confirm their understanding of the study procedures. The study was conducted between March 2016 and June 2016.

We retrospectively screened medical records of patients who had been followed with a diagnosis of CSCR in the Ophthalmology Department of Antalya Education and Research Hospital. Overall, 87 patients fulfilling inclusion criteria and 320 age- and sex-matched healthy individuals as controls were included in the study. Inclusion criteria were as follows:

1. No known comorbid disease.

2. No smoking or alcohol consumption.

3. No use of steroid and/or immunosuppressive medication at the time of laboratory evaluations.
4. No clinical findings of infection at the time of laboratory evaluations.

5. Volunteering to participate.

Patients were excluded if there was no clear data regarding inclusion criteria. Patients with incomplete data were also excluded. The CSCR patients fulfilling inclusion criteria $(\mathrm{n}=87$ ) were classified into 2 groups as acute CSCR (group 1) and chronic CSCR (group 2). Patients presenting with visual acuity loss and visual symptoms, such as micropsia, metamorphopsia, chromatopsia, and central scotomata within 3 months together with neurosensorial retinal detachment on fundoscopy and angiographic focal leakage (ink-plot and smoke stack leakage patterns) were defined as acute CSCR. Patients with visual symptoms for $>3$ months or recurrent symptoms together with focal, diffuse, or mottled leakage during angiography were defined as having chronic CSCR. In all patients, demographic data, disease characteristics, and laboratory results were extracted from patient files. In all patients and controls, $\mathrm{CBC}$, erythrocyte sedimentation rate (ESR), C-reactive protein (CRP), MPV, and NLR values were recorded onto a data sheet. CBC was studied by CellDyn 3700 hematology analyzer using a laser and impedance method, whereas CRP was measured by ARCHITECT Ci4100 analyzer using immunoturbidimetric method. ESR was measured by using automated Biomedical Systems in accordance to Westergreen method. ${ }^{44}$

\section{Statistical analysis}

Descriptive statistics are presented as frequency, percent, mean, SD, and median, minimum-maximum (min-max). Fisher's exact test and Pearson chi-square test were used to assess the relationship between categorical variables. The normality was tested by using Shapiro-Wilks test in groups with sample size of $<50$, whereas it was tested by using Kolmogorov-Smirnov test in groups with sample size of $>50$. In the analyses of difference between measurements in 2 groups, Student's $t$-test was used in variables with normal distribution, whereas Mann-Whitney $U$-test was used in variables with skewed data. Kruskal-Wallis test was used in nonparametric comparisons among 3 groups with post hoc Bonferroni-Dunn procedure in variables found to be significant. In the case of normal distribution, analysis of variance test was used for comparisons among 3 groups, whereas Bonferroni test was used for comparisons between 2 groups. Spearman's correlation analysis was used to assess correlations among continuous variables with skewed distribution, whereas Pearson's correlation test was used to assess correlations between continuous variables with normal distribution. 
Independent variables effective in the discrimination between CSCR and control groups, as well as chronic and acute CSCR groups, were tested by using logistic regression analysis. Results are presented by Wald statistics, odds ratio, and 95\% CI. For analyzing differences between two groups, independent samples $t$-test was used for data with normal distribution, whereas Mann-Whitney test was used for data with skewed distribution. Receiver operating characteristic analysis was performed to determine discriminative value of intergroup difference for some variables. A $P$-value $<0.05$ was considered to be statistically significant. All analyses were performed by using SPSS version 22.0.

\section{Results}

Mean age was $47.33 \pm 10.72$ years in group 1 (32 men and 11 women), $48.86 \pm 11.41$ years in group 2 (31 men and 13 women), and $48.86 \pm 10.89$ years in the control group (250 men, 70 women, group 3) $(P=0.804)$. Tables 1 and 2 present baseline characteristics and parameters evaluated in the CSCR group and in all study groups, respectively.

MPV, CRP, ESR, and NLR values were compared among acute CSCR, chronic CSCR, and control groups. On the basis of comparison among 3 groups, significant difference was detected in at least one group for MPV, CRP, and NLR measurements. Post hoc tests were performed to find the origin of difference. It was found that NLR $(P<0.05)$ and CRP $(P<0.05)$ values were found to be higher in the acute CSCR group than in the other groups. MPV value was found to be higher in the chronic CSCR group than in the other groups $(P<0.001)$.

After combining acute CSCR and chronic CSCR groups to create CSCR group, logistic regression analysis was performed to identify independent risk factors effective

Table I Baseline characteristics in CSCR groups

\begin{tabular}{|c|c|c|c|}
\hline Baseline characteristics & $\begin{array}{l}\text { Group I } \\
(n=43)\end{array}$ & $\begin{array}{l}\text { Group } 2 \\
(n=44)\end{array}$ & $P$-value \\
\hline $\mathrm{BMI}\left(\mathrm{kg} / \mathrm{m}^{2}\right)$ & $25.94 \pm 3.09$ & $26.95 \pm 3.87$ & $0.181^{a}$ \\
\hline Length of education (years) & $8(5-16)$ & $8(5-16)$ & $0.773^{b}$ \\
\hline Disease duration (months) & & $18(5-72)$ & \\
\hline Choroidal thickness $(\mu \mathrm{m})$ & $397.8 \pm 119.34$ & $394.95 \pm 94.52$ & $0.908^{a}$ \\
\hline \multicolumn{4}{|l|}{ Marital status } \\
\hline Married, n (\%) & $34(79.1)$ & $38(86.4)$ & $0.368^{c}$ \\
\hline Single, n (\%) & $9(20.9)$ & $6(13.6)$ & \\
\hline Presence of work stress, n (\%) & $23(53.5)$ & $21(47.7)$ & $0.59 I^{c}$ \\
\hline Presence of familial stress, n (\%) & $16(37.2)$ & $18(40.9)$ & $0.724^{c}$ \\
\hline
\end{tabular}

Notes: andependent samples $t$-test with mean $\pm S D$. 'Mann-Whitney $U$-test with median (min-max). 'Pearson chi-square test with $\mathrm{n}$ (\%). Group I: Acute CSCR; Group 2: Chronic CSCR.

Abbreviations: BMI, body mass index; CSCR, central serous chorioretinopathy. in discrimination between CSCR and control groups. The only independent risk factor effective in disease prediction was MPV in the model involving MPV, CRP, and NLR $(P<0.05$, Table 3$)$.

In addition, logistic regression analysis was performed to identify independent risk factors effective in discrimination between acute and chronic CSCR. All variables were found to be independent risk factors effective in identifying chronic CSCR in the model involving MPV, CRP, and NLR $(P<0.05$, Table 3$)$.

According to receiver operator characteristics curve analysis, optimal MPV cut-off value for discrimination of acute CSCR from chronic CSCR was $>8.95$ with sensitivity of $70.5 \%$ and specificity of $72.1 \%$ (area under curve [AUC]: 0.744). Optimal NLR cut-off value for discrimination of chronic CSCR from acute CSCR was $>1.93$ with sensitivity of $75.0 \%$ and specificity of $74.4 \%$ (AUC: 0.799 , Table 4 ).

\section{Discussion}

This study is the only study evaluating both MPV and NLR together in patients with CSCR. Dursun et al assessed MPV in patients with CSCR. ${ }^{29}$ Similarly, MPV value was found to be higher in CSCR patients than controls. In that study, CSCR patients were not stratified as acute or chronic. Thus, the study by Dursun et al did not provide information as to whether MPV differs between acute and chronic CSCR In our study, MPV was found to be higher in patients with chronic CSCR when compared with patients with acute CSCR and controls, whereas NLR was found to be higher in patients with acute CSCR when compared with patients with chronic CSCR and controls.

In recent years, NLR has been widely used to detect inflammation in cardiovascular disease, malignancies, diabetes mellitus, hypertension, and various inflammatory diseases. ${ }^{30-32}$ NLR is considered as a cost-effective, highly valid marker for systemic inflammation. ${ }^{31}$

In several studies, it was suggested that NLR is associated with endothelial dysfunction in addition to inflammation. CSCR has long been considered as a vasospastic disorder. ${ }^{33}$ Despite advances in imaging modalities, the pathogenesis of CSCR has not been fully elucidated yet. However, the currently accepted theory for pathogenesis is increased choroidal vascular permeability. ${ }^{33}$ In a study aiming to assess choroidal circulation, it was observed that there were dilated capillaries and dilated draining venules in one or more choroidal lobules following delayed, localized arterial filling. ${ }^{8}$ These findings can explain choroidal hyperpermeability in the injured retinal pigment epithelium (RPE) area. These observations can be 
Table 2 Baseline characteristics and parameters evaluated in all study groups

\begin{tabular}{|c|c|c|c|c|}
\hline & $\begin{array}{l}\text { Group I } \\
(n=43)\end{array}$ & $\begin{array}{l}\text { Group } 2 \\
(n=44)\end{array}$ & $\begin{array}{l}\text { Group } 3 \\
(n=320)\end{array}$ & $P$-value \\
\hline Age (years) & $47.33 \pm 10.72$ & $48.86 \pm|I .4|$ & $48.21 \pm 10.89$ & $0.804^{\ddagger}$ \\
\hline Male, n (\%) & $32(74.4)$ & $31(70.5)$ & $250(78.1)$ & $0.485^{*}$ \\
\hline MPV (f/L) & $8.58 \pm\left. 0.9\right|^{a}$ & $9.53 \pm 1.17^{b}$ & $8.56 \pm 0.92^{\mathrm{a}}$ & $<0.00 \mathrm{I}^{\ddagger}$ \\
\hline CRP (mg/L) & $4(3-5.7)^{\mathrm{a}}$ & $3.5(2-5)^{\mathrm{b}}$ & $3.8(2-5.7)^{\mathrm{a}}$ & $0.00 I^{\#}$ \\
\hline $\operatorname{ESR}(\mathrm{mm} / \mathrm{h})$ & $|0.93 \pm 4.2|$ & $|0.4| \pm 4.5 \mid$ & $10.83 \pm 4.38$ & $0.817^{\ddagger}$ \\
\hline NLR & $2.32(1.13-6.27)^{\mathrm{a}}$ & I.6 $(0.57-3.5)^{\mathrm{b}}$ & $\mathrm{I} .72(0.78-1 \mathrm{I} .25)^{\mathrm{b}}$ & $<0.00 I^{\#}$ \\
\hline
\end{tabular}

Notes: ${ }^{\ddagger}$ Analysis of variance with mean \pm SD. *Pearson chi-square test with $\mathrm{n}(\%)$. ${ }^{\sharp}$ Kruskal-Wallis with median (min-max). ${ }^{\mathrm{a}, \mathrm{b}}$ Indicate inter-group differences. Group I: Acute CSCR; Group 2: Chronic CSCR; Group 3: Control.

Abbreviations: CRP, C-reactive protein (normal range: 0-5 mg/L); CSCR, central serous chorioretinopathy; ESR, erythrocyte sedimentation rate (normal range: 0-20 mm/h); MPV, mean platelet volume (normal range: 7.4-40.4 f/L); NLR, neutrophil/lymphocyte ratio.

due to localized lobular inflammation. Moreover, alterations in choroidal circulation, regardless of cause, may cause choroidal ischemia. In one study, endothelin-1 levels were found to be higher in CSCR patients than controls. ${ }^{34}$ Although the pathogenesis of CSCR has not been clearly understood, all these findings indicate a choroidal endothelial dysfunction in CSCR. In our previous study, assessing nailfold videocapillaroscopy findings in patients with CSCR, it was found that pathological patterns, suggestive for microangiopathy, were increased in CSCR patients. ${ }^{35}$ In this study, elevated NLR in patients with acute CSCR could be a marker of endothelial dysfunction. However, further studies with larger sample size are needed to elucidate this issue.

In our study, no significant difference was detected in NLR between chronic CSCR and control groups, whereas NLR was found to be higher in patients with acute CSCR when compared with chronic CSCR and control groups. As mentioned above, the findings observed by Prunte and Flammer could be due to localized lobular inflammation. ${ }^{8}$ Similarly, abnormality in neutrophil chemotaxis may be present in acute CSCR. In the logistic regression analysis of risk factors to identify acute and chronic disease, NLR was found to be significant ( $P=0.017$; OR: 0.295; 95\% CI: 0.108-0.806).

However, there is no data about this issue in the literature; thus, it is impossible to make a comparison. On the basis of our findings, it seems that neutrophils are key cells involved in acute CSCR. We think that NLR can be used as a marker of disease activation in patients with CSCR. However, further studies with larger sample size are needed to elucidate this issue. Similarly, CRP was higher in patients with acute CSCR compared to those with chronic CSCR, although CRP levels obtained were within normal range. In the logistic regression analysis of independent risk factors to identify chronic disease, CRP was found to be significant ( $P=0.010$; OR: 0.326; 95\% CI: 0.139-0.767). Currently, there is not sufficient data on the role of inflammation in the pathogenesis of CSCR, or defining CSCR as a systemic disease. This issue remains unclear as yet. Given our findings, we think that using more sensitive inflammatory markers will be helpful to clarify this issue. The elevated NLR and CRP could be indicators for the presence of subclinical inflammation in acute CSCR. Cortisol is the best known risk factor among those indicated in the pathogenesis of CSCR. ${ }^{36}$ It is well-known that cortisol has lymphopenic effect. Thus, elevated NLR in acute CSCR may be due to 2 causes including the role of neutrophils in the pathogenesis of acute CSCR and steroidrelated lymphopenia.

It is well-known that inflammation stimulates platelets which are known to have a pivotal role in hemostasis. However in recent years, it was shown that platelets can behave as inflammatory cells as they can undergo chemotaxis and release various chemokines and cytokines.

Table 3 Logistic regression analysis of independent risk factors for identification of chronic disease

\begin{tabular}{|c|c|c|c|c|c|c|c|c|c|c|}
\hline \multirow[t]{3}{*}{ Variables } & \multicolumn{5}{|c|}{ CSCR Group 3} & \multicolumn{5}{|c|}{ Group 2 Group I } \\
\hline & \multirow[t]{2}{*}{ Wald } & \multirow[t]{2}{*}{$P$-value } & \multirow[t]{2}{*}{ OR } & \multicolumn{2}{|c|}{ 95\% $\mathrm{Cl}$ for $\mathrm{OR}$} & \multirow[t]{2}{*}{ Wald } & \multirow[t]{2}{*}{$P$-value } & \multirow[t]{2}{*}{ OR } & \multicolumn{2}{|c|}{ 95\% Cl for OR } \\
\hline & & & & Lower & Upper & & & & Lower & Upper \\
\hline MPV & 16.602 & $<0.001$ & 1.657 & 1.300 & 2.112 & 6.221 & 0.013 & 1.999 & 1.160 & 3.445 \\
\hline CRP & 0.152 & 0.696 & 1.069 & 0.763 & 1.498 & 6.600 & 0.010 & 0.326 & 0.139 & 0.767 \\
\hline NLR & 1.880 & 0.170 & I.148 & 0.942 & 1.399 & 5.67 & 0.017 & 0.295 & 0.108 & 0.806 \\
\hline
\end{tabular}

Notes: Group I: Acute CSCR; Group 2: Chronic CSCR; Group 3: Control.

Abbreviations: CRP, C-reactive protein; CSCR, central serous chorioretinopathy; MPV, mean platelet volume; NLR, neutrophil/lymphocyte ratio; OR, odds ratio. 
Table 4 Results of ROC analyses of NLR and MPV in acute and chronic CSCR groups

\begin{tabular}{lllll}
\hline & \multicolumn{4}{l}{ Groups I and 2 } \\
\cline { 2 - 5 } & AUC & Cutoff & Sensitivity & Specificity \\
\hline MPV & 0.744 & 8.95 & 0.705 & 0.721 \\
NLR & 0.799 & 1.93 & 0.750 & 0.744 \\
\hline
\end{tabular}

Notes: Group I: Acute CSCR; Group 2: Chronic CSCR.

Abbreviations: AUC, area under curve; CSCR, central serous chorioretinopathy; MPV, mean platelet volume; NLR, neutrophil/lymphocyte ratio; ROC, receiver operating characteristic.

MPV, which is an indicator of platelet activation, is a simple tool that can be readily measured by hematology analyzers. It has been shown that increased platelet size caused by platelet activation is associated with several inflammatory diseases. ${ }^{19,20,22,23,26}$ In our study, MPV was found to be higher in patients with chronic CSCR when compared with those with acute CSCR and controls.

There was no significant difference between acute CSCR and control groups. In the logistic regression analysis of independent risk factors to identify chronic disease, MPV was found to be significant ( $P=0.013$; OR: 1.999 ; 95\% CI: $1.160-3.445$ ).

Platelets play a pivotal role in the pathogenesis of thrombosis; thus, indirect information can be gathered by laboratory tests analyzing platelet function. Evaluation of MPV value is a way to quantify platelet dysfunction. MPV value measures platelet size and activity. In previous studies, it has been reported that there are correlations between increased MPV value and several thrombotic diseases including deep vein thrombosis, acute myocardial infarction, or cerebrovascular events. ${ }^{37,38}$ These clinical consequences related to high MPV value were explained by the fact that larger platelets are more reactive and tend to aggregate, resulting in endothelial dysfunction. ${ }^{37-41}$ In addition, larger platelets have the capability to store and release higher amounts of $\beta$-thrombogobulin and thromboxane A2. ${ }^{41,42}$ There are several hypotheses attempting to explain CSCR pathogenesis. This is due to the challenge in understanding the etiology of the disease and interest of researchers on this topic. It is known that choroidal permeability is increased in CSCR. One of the hypotheses attempting to explain choroidal hyperpermeability was proposed by Ciardella et al. ${ }^{43}$ Ciardella suggested that prolonged circulatory disruption in choriocapillaris microcirculation causes fluid leakage under the retinal pigment epithelium. Similarly, it is thought that glucocorticoids involved in the pathogenesis of CSCR can cause choroidal hypoperfusion by enhancing blood clotting. ${ }^{5}$ However, all CSCR patients develop chronic disease. Persistence of circulatory abnormalities plays an important role in the progression of chronic CSCR. Higher MPV value in the chronic CSCR group than in the acute CSCR and control groups may be an indicator for the persistence of circulatory abnormality related to microthrombosis. MPV measurement is considered as a cost-effective, routine laboratory evaluation for the demonstration of endothelial dysfunction.

This is the first study in this field. However, there are some limitations of our study. First, sample size should be larger. In addition, by a prospective design, MPV and NLR values at diagnosis and after resolution could be compared in patients with acute CSCR. Due to its retrospective nature, we used ESR and CRP as acute phase reactants. Larger, prospective studies using more sensitive markers of inflammation (hs-CRP) will be more reliable to elucidate this topic.

\section{Conclusion}

It was found that NLR was higher in patients with acute CSCR when compared with chronic CSCR and control groups, whereas MPV was higher in patients with chronic CSCR when compared with acute CSCR and controls. It seems that neutrophils play a major role in acute CSCR, whereas platelets are involved in progression to chronic CSCR. Larger, prospective studies are needed on this topic.

\section{Author contributions}

All authors contributed toward data analysis, drafting, and critically revising the paper, and agree to be accountable for all aspects of the work.

\section{Disclosure}

The authors report no conflicts of interest in this work.

\section{References}

1. Jung SH, Kim KA, Sohn SW, Yang SJ. Cytokine levels of the aqueous humour in central serous chorioretinopathy. Clin Exp Optom. 2014; 97(3):264-269.

2. Akyol M, Erol MK, Ozdemir O, et al. A novel mutation of sgk-1 gene in central serous chorioretinopathy. Int J Ophthalmol. 2015;8(1):23-28.

3. de Carlo TE, Rosenblatt A, Goldstein M, Baumal CR, Loewenstein A, Duker JS. Vascularization of irregular retinal pigment epithelial detachments in chronic central serous chorioretinopathy evaluated with OCT angiography. Ophthalmic Surg Lasers Imaging Retina. 2016;47(2): 128-133.

4. Yannuzzi LA. Type a behavior and central serous chorioretinopathy. Retina. 2012;32(Suppl 1):709.

5. Liew G, Quin G, Gillies M, Fraser-Bell S. Central serous chorioretinopathy: a review of epidemiology and pathophysiology. Clin Experiment Ophthalmol. 2013;41(2):201-214.

6. Nicholson B, Noble J, Forooghian F, Meyerle C. Central serous chorioretinopathy: update on pathophysiology and treatment. Surv Ophthalmol. 2013;58(2):103-126.

7. Guyer DR, Yannuzzi LA, Slakter JS, Sorenson JA, Ho A, Orlock D. Digital indocyanine green videoangiography of central serous chorioretinopathy. Arch Ophthalmol. 1994;112(8):1057-1062. 
8. Prunte C, Flammer J. [Circulatory disorders of the choroid in patients with central serious chorioretinopathy.] Klin Monbl Augenheilkd. 1996; 208(5):337-339. German.

9. Wang M, Munch IC, Hasler PW, Prunte C, Larsen M. Central serous chorioretinopathy. Acta Ophthalmol. 2008;86:126-145.

10. Taub DD, Anver M, Oppenheim JJ, Longo DL, Murphy WJ. T lymphocyte recruitment by interleukin-8(IL-8). IL-8-induced degranulation of neutrophils releases potent chemoattractants for human T lymphocytes both in vitro and in vivo. J Clin Invest. 1996;97(8):1931-1941.

11. Funatsu H, Yamashita H, Ikeda T, Mimura T, Eguchi S, Hori S. Vitreous levels of interleukin- 6 and vascular endothelial growth factor are related to diabetic macular edema. Ophthalmology. 2003;110(9):1690-1696.

12. Koch AE, Polverini PJ, Kunkel SL, et al. Interleukin-8 as a macrophage-derived mediator of angiogenesis. Science. 1992;258(5089): 1798-1801.

13. Shin MC, Lim JW. Concentration of cytokines in the aqueous humor of patients with central serous chorioretinopathy. Retina. 2011;31(9): 1937-1943.

14. Lim JW, Kim MU, Shin MC. Aqueous humor and plasma levels of vascular endothelial growth factor and interleukin- 8 in patients with central serous chorioretinopathy. Retina. 2010;30(9):1465-1471.

15. Moschos MM, Gazouli M, Gatzioufas Z, et al. Prevalence of the complement factor $\mathrm{H}$ and GSTM1 genes polymorphisms in patients with central serous chorioretinopathy. Retina. 2016;36(2):402-407.

16. Yavaş GF, Küsbeci T, Kaşikci M, et al. Obstructive sleep apnea in patients with central serous chorioretinopathy. Curr Eye Res. 2014;39(1): 88-92.

17. Iijima H, Iida T, Murayama K, Imai M, Gohdo T. Plasminogen activator inhibitor 1 in central serous chorioretinopathy. Am J Ophthalmol. 1999; 127(4):477-478

18. Sogutlu Sari E, Yazici A, Eser B, et al. The prevalence of $4 \mathrm{G} / 5 \mathrm{G}$ polymorphism of plasminogen activator inhibitor-1(PAI-1) gene in central serous chorioretinopathy and its association with plasma PAI-1 levels. Cutan Ocul Toxicol. 2014;33(4):270-274.

19. Tuncel T, Uysal P, Hocaoglu AB, Erge DO, Karaman O, Uzuner N. Change of mean platelet volume values in asthmatic children as an inflammatory marker. Allergol Immunopathol (Madr). 2012;40(2): 104-107.

20. Mimidis K, Papadopoulos V, Kotsianidis J, et al. Alterations of platelet function, number and indexes during acute pancreatitis. Pancreatology. 2004;4(1):22-27.

21. Zahorec R. [Ratio of neutrophil to lymphocyte counts - rapid and simple parameter of systemic inflammation and stress in critically ill.] Bratisl Lek Listy. 2001;102(1):5-14. Slovak.

22. Kisacik B, Tufan A, Kalyoncu U, et al. Mean platelet volume (MPV) as an inflammatory marker in ankylosing spondylitis and rheumatoid arthritis. Joint Bone Spine. 2008;75(3):291-294.

23. Endler G, Klimesch A, Sunder-Plassmann H, et al. Mean platelet volume is an independent risk factor for myocardial infarction but not for coronary artery disease. Br J Haematol. 2002;117(2):399-404.

24. Martin JF, Bath PM, Burr ML. Influence of platelet size on outcome after myocardial infarction. Lancet. 1991;338(8780):1409-1411.

25. Van der Loo B, Martin JF. A role for changes in platelet production in the cause of acute coronary syndromes. Arterioscler Thromb Vasc Biol. 1999;19(3):672-679.
26. Huczek Z, Kochman J, Filipiak KJ, et al. Mean platelet volume on admission predicts impaired reperfusion and long-term mortality in acute myocardial infarction treated with primary percutaneous coronary intervention. J Am Coll Cardiol. 2005;46(2):284-290.

27. Biyik M, Ucar R, Solak Y, et al. Blood neutrophil-to-lymphocyte ratio independently predicts survival in patients with liver cirrhosis. Eur $J$ Gastroenterol Hepatol. 2013;25(4):435-441.

28. Tasoglu I, Sert D, Colak N, Uzun A, Songur M, Ecevit A. Neutrophillymphocyte ratio and the platelet-lymphocyte ratio predict the limb survival in critical limb ischemia. Clin Appl Thromb Hemost. 2014;20(6): 645-650.

29. Dursun A, Toker MI, Ozec AV, et al. Relationship between mean platelet volume and central serous chorioretinopathy. Int Ophthalmol. 2017;37(1):119-124.

30. Fici F, Celik T, Balta S, et al. Comparative effects of nebivolol and metoprolol on red cell distribution width and neutrophil/lymphocyte ratio in patients with newly diagnosed essential hypertension. J Cardiovasc Pharmacol. 2013;62(4):388-393.

31. Balta S, Demirkol S, Unlu M, Arslan Z, Celik T. Neutrophil to lymphocyte ratio may be predict of mortality in all conditions. Br J Cancer. 2013;109(12):3125-3126.

32. Balta S, Demirkol S, Celik T, et al. Association between coronary artery ectasia and neutrophil-lymphocyte ratio. Angiology. 2013;64(8): 627-632.

33. Liegl R, Ulbig MW. Central serous chorioretinopathy. Ophthalmologica. 2014;232(2):65-76.

34. Prunte C, Haufschild T, Schroeder B, Flammer J. Microvascular dysregulation in central serous chorioretinopathy. Invest Ophthalmol Vis Sci. 2003;44:4308.

35. Erol MK, Balkarli A, Toslak D, et al. Evaluation of nailfold videocapillaroscopy in central serous chorioretinopathy. Graefe's Arch Clin Exp Ophthalmol. 2016;254(10):1889-1896.

36. Garg SP, Dada T, Talwar D, Biswas NR. Endogenous cortisol profile in patients with central serous chorioretinopathy. Br J Ophthalmol. 1997; 81(11):962-964.

37. Vagdatli E, Gounari E, Lazaridou E, Katsibourlia E, Tsikopoulou F, Labrianou I. Platelet distribution width: a simple, practical and specific marker of activation of coagulation. Hippokratia. 2010;14(1):28-32.

38. Sahin A, Sahin M, Yuksel H, et al. The mean platelet volume in patients with retinal vein occlusion. J Ophthalmol. 2013;2013:236371.

39. Haznedaroglu IC, Ozcebe OI, Ozdemir O, Celik I, Dündar SV, Kirazli S. Impaired haemostatic kinetics and endothelial function in Behcet's disease. J Intern Med. 1996;240(4):181-187.

40. Jakubowski JA, Thompson CB, Vaillancourt R, Valeri CR, Deykin D. Arachidonic acid metabolism by platelets of differing size. Br J Haematol. 1983;53(3):503-511.

41. Giles H, Smith RE, Martin JF. Platelet glycoprotein IIb-IIIa and size are increased in acute myocardial infarction. Eur J Clin Invest. 1994;24(1): 69-72.

42. Haver VM, Gear AR. Functional fractionation of platelets. J Lab Clin Med. 1981;97(2):187-204.

43. Ciardella AP, Guyer DR, Spitznas M. Central serous chorioretinopathy. In: Retina, Ryan S. St Louis, MO: Mosby; 2001:1153-1181.

44. Gilmour D, Sykes AJ. Westergren and Wintrobe Methods of Estimating E.S.R. Compared. Br Med J. 1951;2(4746):1496-1497.
Therapeutics and Clinical Risk Management

\section{Publish your work in this journal}

Therapeutics and Clinical Risk Management is an international, peerreviewed journal of clinical therapeutics and risk management, focusing on concise rapid reporting of clinical studies in all therapeutic areas, outcomes, safety, and programs for the effective, safe, and sustained use of medicines. This journal is indexed on PubMed Central, CAS,

\section{Dovepress}

EMBase, Scopus and the Elsevier Bibliographic databases. The manuscript management system is completely online and includes a very quick and fair peer-review system, which is all easy to use. Visit http://www.dovepress.com/testimonials.php to read real quotes from published authors. 